FN-0763-AD

\title{
DECOHERENCE AND LANDAU-DAMPING
}

\author{
K.Y. Ng \\ Fermi National Accelerator Laboratory, ${ }^{*}$ P.O. Box 500, Batavia, IL 60510
}

(July, 2005)

\begin{abstract}
The terminologies, decoherence and Landau damping, are often used concerning the damping of a collective instability. This article revisits the difference and relation between decoherence and Landau damping. A model is given to demonstrate how Landau damping affects the rate of damping coming from decoherence.
\end{abstract}

*Operated by the Universities Research Association, Inc., under contract with the U.S. Department of Energy. 


\section{Introduction}

Decoherence and Landau damping are two commonly quoted terminologies when the curbing an instability is addressed. To stop a transverse instability with growth rate $\tau^{-1}$, for example, the spread in betatron frequency required is $\Delta \omega_{\beta} \approx \tau^{-1}$, roughly the same both for decoherence and Landau damping. We want to ask: are decoherence and Landau damping the same thing? If they are not the same, we should compute the damping rate from decoherence and the damping rate from Landau damping separately, so that the sum of the two damping rates then becomes the true damping rate of the beam for that instability. If they are the same, this summation will lead to double counting. Thus it is very important to understand what is decoherence and what is Landau damping.

In Sec. 2, we review the concepts of decoherence and Landau damping in an ensemble of oscillators. The study is next extended in Sec. 3 to a beam of charged particles in an accelerator vacuum chamber. We will see that decoherence and Landau damping, though very different in concept, are inseparable. Although decoherence is not Landau damping, however, the Landau-damping does include decoherence. An example will be given to illustrate how Landau damping influences decoherence. Sec. 4 is devoted to study the decoherence in the longitudinal phase space. Finally in Sec. 5, conclusions are given. There have been quite a number of good papers written on this subject by, for example, Hereward, [2] Hofmann, [3] and Chao. [4] Some discussions in these articles will be used in this paper.

\section{An Ensemble of Oscillators}

\section{$2.1 \quad$ Decoherence}

Let us consider first an ensemble of oscillators, each having slightly different angular oscillation frequencies $\omega$. If all oscillators are to have the same initial conditions, the motion of the oscillators becomes out of phase as time progresses and the average displacement of the ensemble decays. This is known as decoherence.

The process can be described mathematically. An oscillator with displacement $y$ and angular frequency $\omega$ satisfies the equation of motion

$$
\ddot{y}+\omega^{2} y=0
$$

where the overdot represents derivative with respect to time. The most general solution is

$$
y(t)=y_{0} \cos \omega t+\dot{y}_{0} \frac{\sin \omega t}{\omega},
$$

where $y_{0}$ and $\dot{y}_{0}$ are, respectively, the initial values of $y$ and $\dot{y}$ at time $t=0$. If all the oscillators start with the same initial conditions, either $y_{0} \neq 0$ but $\dot{y}_{0}=0$ or $\dot{y}_{0} \neq 0$ and 
$y_{0}=0$, the average displacement of the ensemble becomes, as time evolves,

$$
\langle y(t)\rangle=y_{0} H(t) \int d \omega \cos \omega t \rho(\omega), \quad \dot{y}_{0}=0,
$$

or

$$
\langle\dot{y}(t)\rangle=\dot{y}_{0} H(t) \int d \omega \cos \omega t \rho(\omega), \quad y_{0}=0,
$$

where $H(t)$ is the Heaviside step function and $\rho(\omega)$ is the distribution of oscillation frequency in the ensemble of oscillators, which is normalized according to

$$
\int_{-\infty}^{\infty} \rho(\omega) d \omega=1
$$

We identify

$$
G(t)=H(t) \int d \omega \cos \omega t \rho(\omega),
$$

and call it the shock response function, which is always real and vanishes when $t<0$. It represents the time evolution of the average displacement of the ensemble of oscillators after it is displaced by one unit. As an example, take the Lorentz distribution with half-widthat-half-maximum $\Delta \omega$,

$$
\rho(\omega)=\frac{\Delta \omega}{\pi} \frac{1}{(\omega-\bar{\omega})^{2}+(\Delta \omega)^{2}},
$$

where $\bar{\omega}$ is the mean angular frequency of the ensemble. The shock response function is easily found to be

$$
G(t)=H(t) \mathcal{R} e e^{i(\bar{\omega}+i \Delta \omega) t}=H(t) e^{-\Delta \omega t} \cos \bar{\omega} t,
$$

and is depicted in Fig. 1. We see clearly that the average displacement of the oscillators

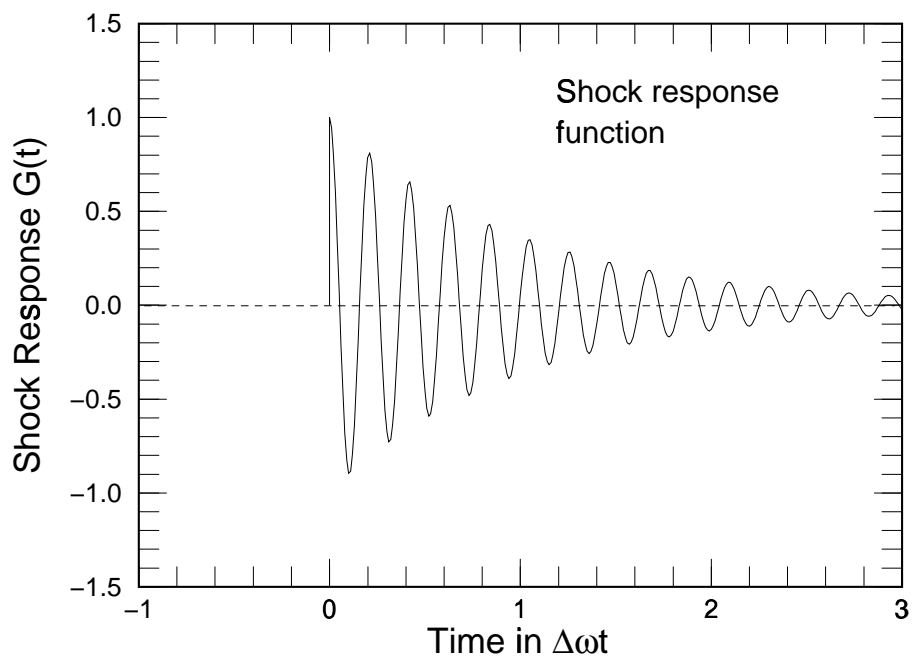

Figure 1: Plots showing the shock response function $G(t)$ for Lorentz frequency distribution. 
decays with the $e$-folding time $1 / \Delta \omega$. In other words, if there is a collective transverse instability, with a growth rate $\tau^{-1} \approx \Delta \omega$, the instability will not have been able to materialize.

We give this process the name kinematic decoherence. It is so called because only kinematics is involved and the equation of motion of each oscillator involves only its own intrinsic frequency, which may also be amplitude dependent. A driving force either external or arising from other oscillators, if present, will modify the decoherence.

\subsection{Landau Damping}

Now an harmonic force of amplitude $A$ and angular frequency $\Omega$ is exerted onto the ensemble of oscillators. The equation describing the displacement of one oscillator becomes

$$
\ddot{y}+\omega^{2} y=A \cos \Omega t \text {. }
$$

The most general solution is

$$
y(t)=y_{0} \cos \omega t+\dot{y}_{0} \frac{\sin \omega t}{\omega}+\frac{A}{\omega^{2}-\Omega^{2}}[\cos \Omega t-\cos \omega t] .
$$

The first two terms are due to a shock or $\delta$-pulse excitation, which have been discussed before and has been shown to contribute to kinematic decoherence. As a result, these terms will be dropped here in the present discussion. The third term is the response to the harmonic force. Notice that this term is well-behaved even at $\omega=\Omega$. For the whole ensemble, the displacement of the center-of-mass is

$$
\langle y(t)\rangle=A \int_{-\infty}^{\infty} d \omega \frac{\rho(\omega)}{\omega^{2}-\Omega^{2}}[\cos \Omega t-\cos \omega t] .
$$

The distribution $\rho(\omega)$ is assumed to be a narrow one centered at angular frequency $\bar{\omega}$. For simplicity, let us assume that this distribution does not peak at any other frequency. ${ }^{\dagger}$ In order to drive this ensemble of oscillators, the driving frequency must also be close to this center frequency, or $\Omega \approx \bar{\omega}$. We can therefore perform the expansion $\omega=\Omega+(\omega-\Omega)$, and Eq. (2.11) can be approximated by

$$
\langle y(t)\rangle=\frac{A}{2 \bar{\omega}}\left[\cos \Omega t \int_{-\infty}^{\infty} d \omega \rho(\omega) \frac{1-\cos (\omega-\Omega) t}{\omega-\Omega}+\sin \Omega t \int_{-\infty}^{\infty} d \omega \rho(\omega) \frac{\sin (\omega-\Omega) t}{\omega-\Omega}\right] .
$$

${ }^{\dagger}$ We may also assume the distribution to peak at both $\pm \bar{\omega}$ with the symmetry property $\rho(\omega)=\rho(-\omega)$. Then we obtain instead

$$
\begin{aligned}
\langle y(t)\rangle & =\frac{A}{2 \Omega}\left[\int_{-\infty}^{\infty} d \omega \frac{\rho(\omega)}{\omega-\Omega}-\int_{-\infty}^{\infty} d \omega \frac{\rho(\omega)}{\omega+\Omega}\right][\cos \Omega t-\cos \omega t] \\
& =\frac{A}{\Omega} \int_{-\infty}^{\infty} d \omega \frac{\rho(\omega)}{\omega-\Omega}[\cos \Omega t-\cos \omega t] .
\end{aligned}
$$

However, the distributions commonly used, for example the Gaussian $\rho(\omega)=\exp \left[-(\omega-\bar{\omega})^{2}\right] /\left(2 \sigma^{2}\right)$, do not possess this symmetry property. 
Notice that the fast-oscillating term of angular frequency $\Omega$ and the slow-oscillating envelopelike terms with angular frequency $\omega-\Omega$ have been separated. We also see a part, the $\cos \Omega t$ term, that is not driven in phase $e^{\ddagger}$ by the force, and the other part, the sin $\Omega t$ term, that is driven in phase by the force. It is interesting to see the asymptotic behavior of the average displacement of the ensemble. We find that as $t \gg 1 / \Delta \omega$, where $\Delta \omega$ is a measure of the width of the frequency distribution $\rho(\omega)$, all the transients die, leaving us with (see Appendix)

$$
\langle y(t)\rangle=\frac{A}{2 \bar{\omega}}\left[\cos \Omega t \wp \int_{-\infty}^{\infty} d \omega \frac{\rho(\omega)}{\omega-\Omega}+\pi \rho(\Omega) \sin \Omega t\right] .
$$

The term proportional to $\sin \Omega t$ in Eq. (2.13) is driven in phase by the harmonic force, and the oscillators should be absorbing energy. Let us rewrite Eq. (2.11) in the approximation that the frequency distribution $\rho(\omega)$ is narrow around $\bar{\omega}$ :

$$
\langle y(t)\rangle=\frac{A \sin \bar{\omega} t}{\bar{\omega}} \int_{-\infty}^{\infty} d \omega \rho(\omega) \frac{\sin \frac{1}{2}(\omega-\Omega) t}{\omega-\Omega} .
$$

Consider a component corresponding to the frequency $\omega$, its envelope is

$$
\text { Amplitude }(\omega)=\frac{A}{\bar{\omega}} \frac{\sin \frac{1}{2}(\omega-\Omega) t}{\omega-\Omega} .
$$

This means that all oscillators having frequency $\omega$ are excited at $t=0$, increase to a maximum of $A /[\bar{\omega}(\omega-\Omega)]$ at $t \approx \pi /(\omega-\Omega)$, and die down to zero again at $t=2 \pi /(\omega-\Omega)$. Thus, energy is gained but is given back to the ensemble. For $\omega$ closer to $\Omega$, the response amplitude rises to a larger amplitude and the energy is given back to the ensemble at a later time. For those oscillators that have exactly the frequency $\Omega$, the amplitude grows linearly with time and the absorption of energy continues indefinitely. This process of ceasing amplitude growth except for the few oscillators having frequencies very close to $\Omega$ is called Landau damping. An illustration is shown in Fig. 2, where the solid curve shows an oscillator having exactly the same frequency as $\Omega$ and growing linearly, while the dashed curve shows an oscillator with frequency $95 \%$ of $\Omega$ decaying after about ten oscillations. In other words, oscillators with $\omega$ far away from $\Omega$ get excited, but the energy is returned, while those oscillators having $\omega$ close to $\Omega$ are still absorbing energy. Thus, as time progresses, less and less oscillators will continue to absorb energy. As $t \rightarrow \infty$, only oscillators with frequency exactly equal to $\Omega$ will be absorbing energy, and there are only very few oscillators doing this. If there is a limitation to the amplitude such as the vacuum chamber of an accelerator, the process stops when the growing amplitudes hit the limitation. This sets the time limit for Landau damping to stop. The damping process starts when the amplitude of the first oscillator is damped and this time is $t \approx 2 \pi / \Delta \omega$.

\footnotetext{
${ }^{\ddagger}$ Actually, "in phase" here implies the driving force is in phase with the velocity $\dot{x}$.
} 


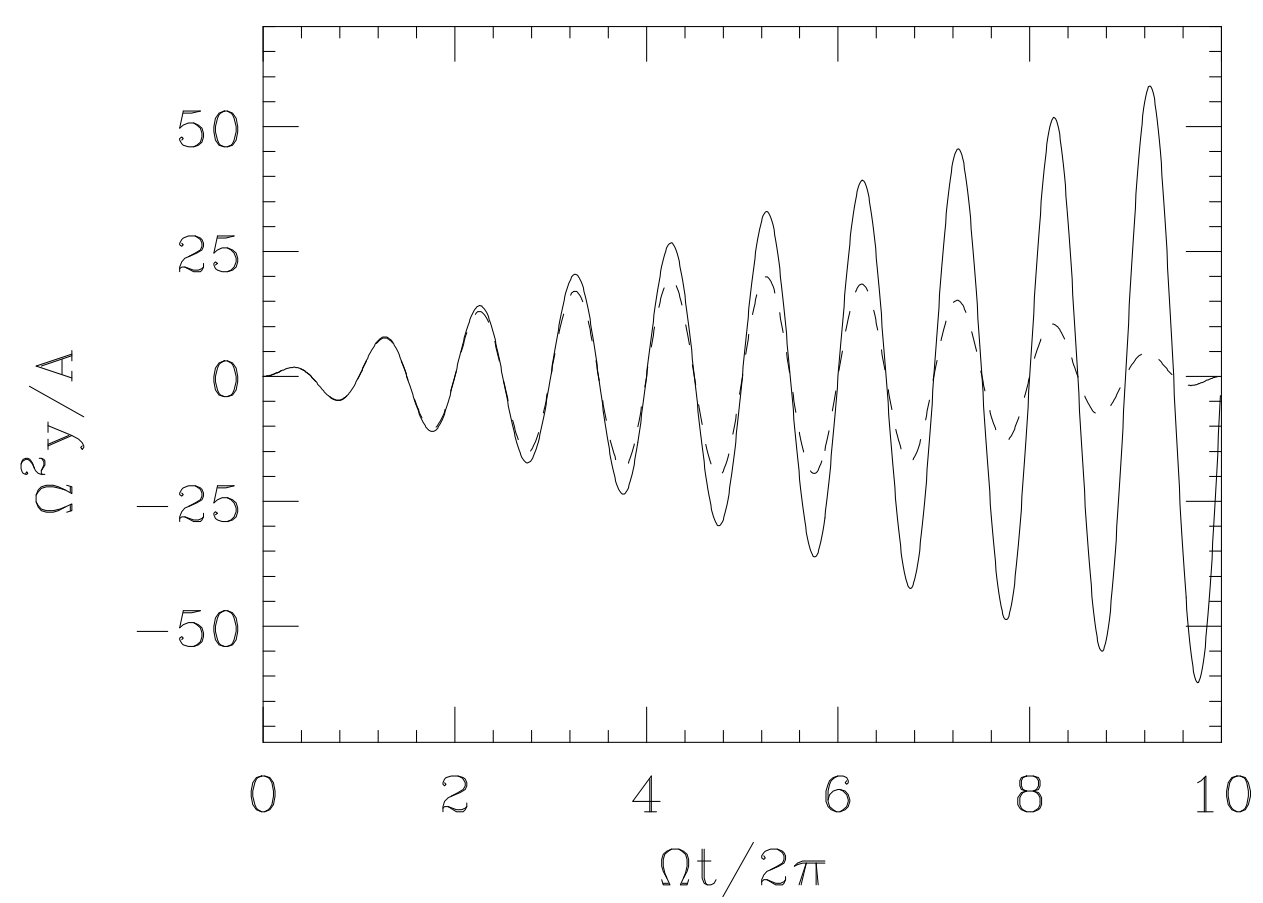

Figure 2: Solid: The response of an oscillator having exactly the same frequency $\Omega$ as the driving force grows linearly in time. Dashes: The response of an oscillator having frequency $95 \%$ of $\Omega$ gives up its energy after about 10 oscillations.

Let us study the energy distribution in the ensemble. The energy is proportional to the square of the amplitude,

$$
[\text { Amplitude }(\omega)]^{2}=\frac{\sin ^{2}(\omega-\Omega) t / 2}{(\omega-\Omega)^{2}}
$$

Therefore the energy of all the oscillators is

$$
\mathcal{E}=\frac{N A^{2}}{\bar{\omega}^{2}} \int_{-\infty}^{\infty} d \omega \rho(\omega) \frac{\sin ^{2}(\omega-\Omega) t / 2}{(\omega-\Omega)^{2}},
$$

where $N$ is the total number of oscillators in the ensemble. Figure. 3 shows the energy distribution at the normalized time $T=\Omega t=150$ as a function of fractional frequency offset $\delta \hat{\omega}=(\omega-\Omega) / \Omega$, where a flat distribution has been assumed for simplicity. It is easy to see all oscillators with frequency offset $|\delta \hat{\omega}|<\pi / T=0.021$ are inside the central area of the plot and these oscillators continue to absorb energy from the harmonic force. On the other hand, those oscillators outside the central area have their amplitude varying sinusoidally with period $\Delta T=\pi / \delta \hat{\omega}$, implying that they gain energy for half a period and give out energy for the other half period. As time progresses the peak of central area, equal to $T^{2}$, becomes taller and taller while its width shrinks. This verifies that more and more oscillators stop gaining energy and the number of oscillators continue gaining energy becomes smaller and 


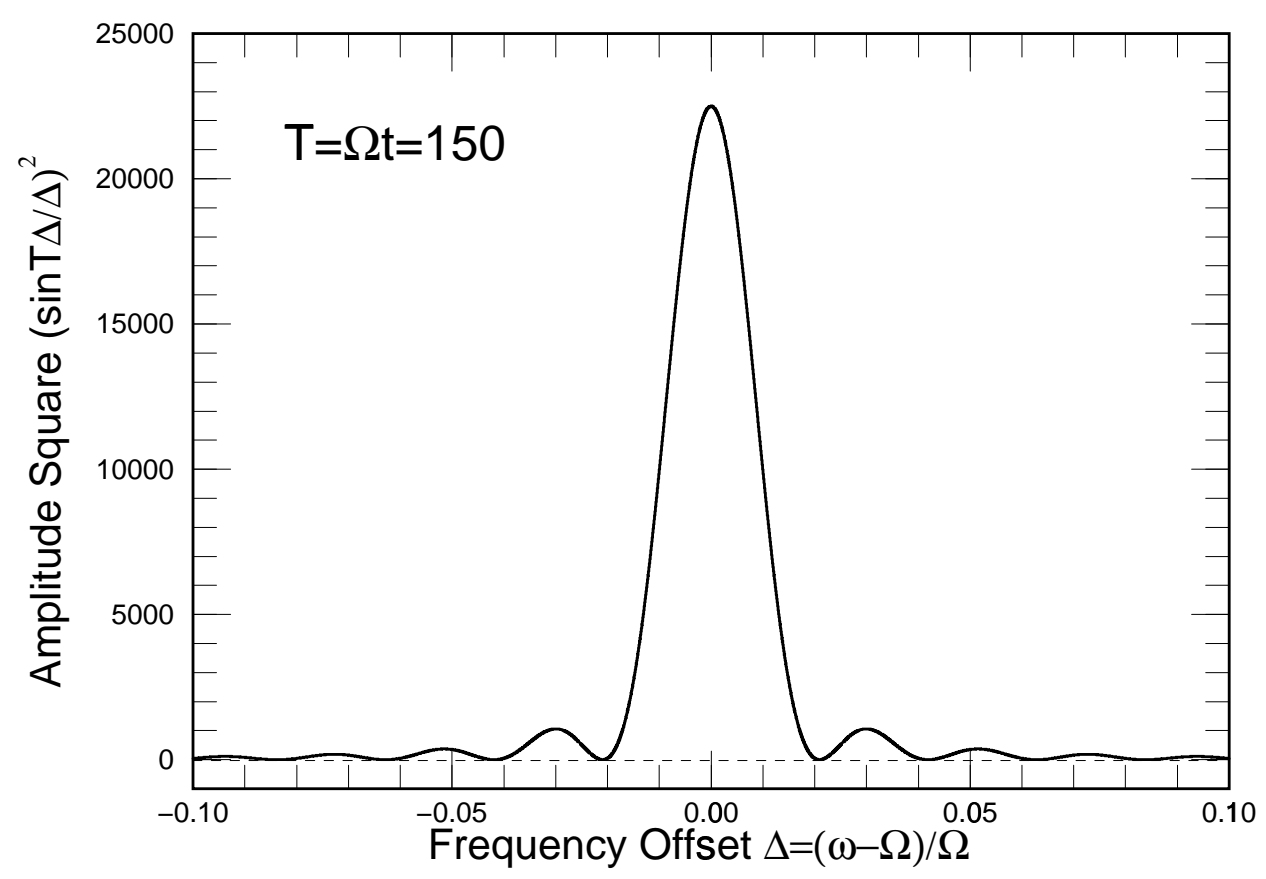

Figure 3: Energy or amplitude squared distribution of the ensemble of oscillators as a function of fraction frequency offset $\delta \hat{\omega}=(\omega-\Omega) / \Omega$ at the normal time $T=\Omega T=150$. Oscillators inside the central area continue to gain energy. Oscillators outside have their amplitude oscillating; they gain energy in a half period $\Delta T=\pi /(2 \delta \hat{\omega})$ and give up energy in the next half period. Uniform distribution in frequency is assumed.

smaller. ${ }^{\S}$ The fraction of energy possessed by oscillator inside the central area is

$$
\frac{\int_{-\pi / T}^{\pi / T} \frac{\sin ^{2} T \delta \hat{\omega}}{\delta \hat{\omega}^{2}} d \delta \hat{\omega}}{\int_{-\infty}^{\infty} \frac{\sin ^{2} T \delta \hat{\omega}}{\delta \hat{\omega}^{2}} d \delta \hat{\omega}}=\frac{2}{\pi} \int_{0}^{\pi} \frac{\sin ^{2} u}{u^{2}} d u=0.903
$$

\footnotetext{
${ }^{\S}$ It is often said that energy is being transferred from oscillators with frequencies $\omega$ far from $\Omega$ to those oscillators with frequencies much closer to $\Omega$. Strictly speaking, this statement is incorrect in this model. From Eq. (2.9), it is clear that the oscillators are driven independently by the harmonic force. Since there is no coupling between the oscillators, there is no way for energy to be transferred from one oscillator to the other. In other words, each oscillator can be studied independently. Because the harmonic force is external, there is no conservation of energy. The harmonic force drives an oscillator into motion with increasing amplitude when it is in phase with the oscillator motion and damps the motion when out of phase. This period of growing amplitude followed by damping is $\pi / \delta \hat{\omega}$. As a result, the average energy of the oscillator under the influence of the force is always the same, although it does oscillate sinusoidally. For an oscillator with frequency very close to $\Omega$, this oscillatory period of the amplitude is very long, or the oscillator continues to absorb energy for a very long time. Since energy is exchanged between the oscillator and the harmonic force, one may say loosely that energy is exchanged between oscillators through the harmonic force. As will be seen below energy exchange between beam particles does occur when they are coupled by the impedance of the vacuum chamber.
} 
which is time independent. This implies that at any instant $90.3 \%$ of the energy reside in oscillators that are absorbing energy continuously and these oscillators because less and less as time progresses.

Since the square of the amplitude always covers an area of $\pi t / 2$, we have

$$
\lim _{t \rightarrow \infty}[\operatorname{Amplitude}(\omega)]^{2}=\lim _{t \rightarrow \infty} \frac{\sin ^{2}(\omega-\Omega) t / 2}{(\omega-\Omega)^{2}}=\frac{\pi t}{2} \delta(\omega-\Omega) .
$$

Thus, at $t \rightarrow \infty$, the steady-state energy of the ensemble is

$$
\mathcal{E}=\frac{\pi}{2} \frac{N A^{2}}{\bar{\omega}^{2}} \rho(\Omega) t
$$

which increases linearly with time, and after an infinitely long time, all this energy goes into those few oscillators having exactly the same frequency as $\Omega$. However, we do see in the asymptotic solution of Eq. (2.14) that $\langle y(t)\rangle$ does not go to infinity. This is not a contradiction, because even when a few oscillators have very large and still growing amplitudes, the centroid will not be affected by very much.

We now see that decoherence is very different from Landau damping. Decoherence is the response of a shock excitation on the ensemble and the mechanism is kinematic in nature. On the other hand, Landau damping is the response of a driving force on the ensemble. At the beginning when the force is exerted, all oscillators are driven in phase and the displacement of the center of the ensemble increases rapidly. However, the motion of more and more oscillators becomes out of phase because of the spread in frequency among the oscillators. The displacement of the center of the ensemble starts decaying. The decay of the displacement of the center is obviously the result of decoherence. However, this is different from the kinematic decoherence after a shock excitation. It is the inability of an harmonic force to excite the ensemble of oscillators coherently. For this reason, we can call Landau damping dynamic decoherence.

\section{Damping of a Beam}

In our study so far, the amplitude $A$ of the driving force is independent of the ensemble of oscillators. For an instability in a particle beam, the situation is slightly different. The driving force comes from the wake fields of the beam particles interacting with the discontinuities of the vacuum chamber, and usually has an amplitude proportional to the center displacement of the beam. When there is a kick to the beam that creates a center displacement $\langle y(0)\rangle$ or a center displacement velocity $\langle\dot{y}(0)\rangle$, a force with amplitude $A \propto\langle y(0)\rangle$ or $\langle\dot{y}(0)\rangle$ is generated and drives the whole system of particles with the coherent frequency $\Omega$. Each frequency component of the beam will receive the amount of response according to Eq. (2.16). Now two things happen. First, the particles give up their excited energy gradually to those particles having frequencies extremely close to $\Omega$, the frequency of the driving force, and the center of 
displacement approaches the transfer function $R(u)$. Second, the center of displacement of the beam starts to decay according to the shock response function $G(t)$. As $\langle y(t)\rangle$ decreases, the driving force decreases also. Finally, the disturbance goes away. This is how Landau damping takes place in a beam. In fact, this process starts whenever the disturbance is of infinitesimal magnitude, implying that any disturbance will be damped as soon as it occurs. We say that there will be enough Landau damping to keep the beam stable. Notice that no frictional force has ever been introduced in the discussion. Thus, there is still conservation of energy in the presence of Landau damping, which merely redistributes energy from waves of one frequency to another.

In case the frequency spread $\Delta \omega$ is very very narrow, it will take $t \approx \pi / \Delta \omega$ for the first wave to surrender its energy to another that has frequency closer to $\Omega$. This duration will be very long. Before this moment arrives, all frequency components continue to receive energy and $\langle x(t)\rangle$ increases and so will be the driving force. This is the picture of how an instability develops when the spread of frequency is not large enough to invoke Landau damping. However, the conservation of energy still holds. The energy that feeds the instability may be extracted from the longitudinal kinetic energy of the beam resulting in a slower speed, or from the rf system that replenishes the beam energy.

Let us consider the transverse betatron motion of a beam of particles. The equation of motion governing the vertical displacement of a beam particle inside a coasting beam is

$$
\ddot{y}+\omega_{\beta}^{2} y=i A\langle y\rangle,
$$

where the wake force on the right is proportional to the vertical displacement of the centerof-mass of the beam and the constant $A$ plays the role of the transverse impedance of the vacuum chamber. More concretely, it can be easily shown that

$$
A=\frac{e I_{0} \beta c^{2} Z_{1}^{\perp}}{2 \pi R E_{0}}
$$

where $I_{0}$ is the beam current, $E_{0}$ the nominal energy of a beam particle, $R$ the mean radius of the ring, and $Z_{1}^{\perp}$ is the transverse impedance of the vacuum chamber, which is considered to be broadband. The overdots on the displacement $y(\theta, t)$ represent the second-order total time derivative

$$
\frac{d^{2}}{d t^{2}}=\left(\frac{\partial}{\partial t}+\omega_{0} \frac{\partial}{\partial \theta}\right)^{2}
$$

with $\theta$ denoting the azimuthal angle around the ring and $\omega_{0}$ is the angular revolution frequency. All the particles are initially at rest with $y=0$ and $\dot{y}=0$. At time $t=0$, a vertical displacement $\left.y(\theta, t)\right|_{\mathrm{t}=0}=\sum_{n} y_{n 0} e^{i n \theta}$ and transverse velocity $\partial y(\theta, t) /\left.\partial t\right|_{\mathrm{t}=0}=\sum_{n} \dot{y}_{n 0} e^{i n \theta}$ are imparted to the beam. We are going to study the time response of the center-of-mass. Let us introduce the double Fourier transform

$$
\tilde{y}_{n}(\omega)=\int_{0}^{2 \pi} \frac{d \theta}{2 \pi} \int_{0}^{\infty} \frac{d t}{2 \pi} y(\theta, t) e^{-i n \theta+i \omega t}
$$


The inverse Fourier transform is given by

$$
y(\theta, t)=\sum_{n=-\infty}^{\infty} \int_{W} \tilde{y}_{n}(\omega) e^{i n \theta-i \omega t} d \omega,
$$

where the contour $W$ is from $-\infty+i \alpha$ to $\infty+i \alpha$ with $\alpha>0$ and chosen in such a way that the contour lies above all singularities of $\tilde{y}(\omega)$ so that causality is preserved. After the Fourier transform, Eq. (3.1) becomes the algebraic equation

$$
-\frac{\dot{y}_{n 0}}{2 \pi}+\frac{i\left(\omega-2 n \omega_{0}\right) y_{n 0}}{2 \pi}+\left(\omega_{\beta}^{2}-\Omega^{2}\right) \tilde{y}_{n}(\omega)=i A\left\langle\tilde{y}_{n}(\omega)\right\rangle
$$

where $\Omega=\omega-n \omega_{0}$. In obtaining Eq, (3.6), we have made the assumption that $\omega$ lies in the upper half-plane so that the evaluation at $t=\infty$ can be performed. For simplicity, we set $\dot{y}_{n 0}=0$ and solve for

$$
\tilde{y}_{n}(\omega)=\frac{i A\langle\tilde{y}(\omega)\rangle}{\omega_{\beta}^{2}-\Omega^{2}}-\frac{i y_{n 0}}{2 \pi} \frac{\omega-2 n \omega_{0}}{\omega_{\beta}^{2}-\Omega^{2}} .
$$

Next integrate both sides with the distribution function $\rho\left(\omega_{\beta}\right)$ to obtain

$$
\left\langle\tilde{y}_{n}(\omega)\right\rangle=\frac{-i y_{n 0}\left(\omega-2 n \omega_{0}\right) \int_{-\infty}^{\infty} \frac{\rho\left(\omega_{\beta}\right)}{\omega_{\beta}^{2}-\Omega^{2}} d \omega_{\beta}}{2 \pi \mathcal{H}(\omega)},
$$

where the function in the denominator is defined as

$$
\mathcal{H}(\omega)=1-i A \int_{-\infty}^{\infty} \frac{\rho\left(\omega_{\beta}\right)}{\omega_{\beta}^{2}-\Omega^{2}} d \omega_{\beta} \quad \text { when } \operatorname{Im} \omega>0 .
$$

It will be convenient to define $\left\langle\tilde{y}_{n}(\omega)\right\rangle$ in the lower $\omega$-plane as well so that contour integration can be performed later to obtain the temporal evolution of the displacement of the centerof-mass. It is easy to see that, as defined in Eq. (3.9), $\mathcal{H}(\omega)$ is discontinuous across the real $\omega$-axis. Analytic continuation from the upper $\omega$-plane to the lower $\omega$-plane can be performed easily by patching the discontinuity. This can be accomplished easily by changing the contour of integration. Following Landau, this analytically-continued function can be written conveniently as [1]

$$
\mathcal{H}(\omega)=1-i A \int_{C} \frac{\rho\left(\omega_{\beta}\right)}{\omega_{\beta}^{2}-\Omega^{2}} d \omega_{\beta},
$$

where the contour $C$ is the path from $\omega_{\beta}=-\infty$ to $\infty$ with the detour of going above the pole at $\omega_{\beta}=-\Omega$ and under the pole at $+\Omega$, as illustrated in Fig. 4 . It is important to note that $\mathcal{H}(\omega)=0$ is just the dispersion relation governing the stability of the beam.

Now transforming back to the time domain, the response of the center-of-mass takes the form,

$$
\langle y(\theta, t)\rangle=\sum_{n=-\infty}^{\infty} y_{n 0} \int_{W} \frac{d \omega}{2 \pi i} e^{i n \theta-i \omega t} \frac{\int_{C} \frac{\left(\omega-2 n \omega_{0}\right) \rho\left(\omega_{\beta}\right)}{\omega_{\beta}^{2}-\Omega^{2}} d \omega_{\beta}}{\mathcal{H}(\omega)} .
$$




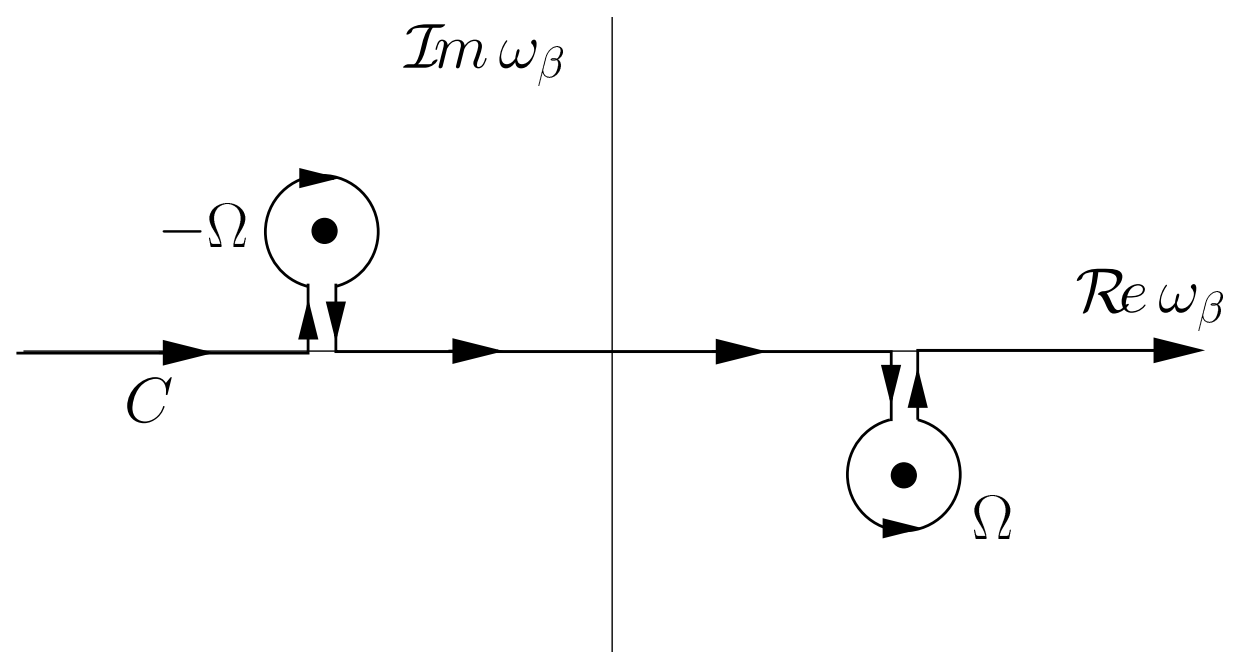

Figure 4: To continue $\mathcal{H}(\omega)$ from the upper half $\omega$-plane to the lower half $\omega$-plane, the path of integration $C$ in the $\omega_{\beta}$-plane must go above the pole $-\Omega$ and under the pole $+\Omega$.

The solution consists of many waves which have frequencies that are the zeroes of $\mathcal{H}(\omega)$. These zeroes are the collective eigen-frequencies of the dispersion relation and therefore of the beam. First, let us consider the situation when there is no coupling impedance or $\mathcal{H}=1$. The integration over $\omega$ is performed first. Remembering that the contour of integration is above all singularities, $\langle y(t)\rangle=0$ is obtained when $t<0$ by completing the upper semi-circle. But for $t>0$, one must complete the lower semi-circle picking up the two poles at $\pm \Omega$ to arrive at

$$
\langle y(\theta, t)\rangle=\sum_{n=-\infty}^{\infty} y_{n 0} e^{i n\left(\theta-\omega_{0} t\right)} \int_{-\infty}^{\infty} d \omega_{\beta} \rho\left(\omega_{\beta}\right)\left(\cos \omega_{\beta} t+\frac{i n \omega_{0}}{\omega_{\beta}} \sin \omega_{\beta} t\right),
$$

which is the decoherence of the center of the beam subject to a sudden harmonic displacement at time $t=0$. If this initial sudden displacement is the same for all beam particles, or $y_{n 0}=y_{0} \delta_{n 0}$, the above simplifies readily to, for $t>0$,

$$
\langle y(\theta, t)\rangle=y_{0} \int_{-\infty}^{\infty} d \omega_{\beta} \rho\left(\omega_{\beta}\right) \cos \omega_{\beta} t
$$

which is just the shock response function as defined in Eq. (2.8).

In the presence of the coupling impedance, $\mathcal{H}$ differs from unity and the response consists of one or more collective waves instead. If the collective effect, driven by the impedance of the vacuum chamber, is large and the spread in betatron frequency is not large enough for damping, there will be a growing collective wave which dominates over all other collective waves in the response $\langle y(t)\rangle$. The response will therefore have large deviation from the shock response function (or from kinematic decoherence). However, if the spread in betatron frequency is large enough so that the collective instability is Landau damped, then the 
collective waves will all be damped, and the response of the center-of-mass will not deviate much from the shock response function. In that sense, we may say Landau damping is not much different from kinematic decoherence. Thus there will not be double counting because the dynamical effect is small. This explains why it is not easy to reveal the impedance effects by monitoring decoherence after a sudden kick of the beam.

An example is Experiment E778 performed at the Fermilab Tevatron at the design era of the Superconducting Super Collider (SSC), where the linear aperture of the lattice was pursued. [5] Since the beam in the Tevatron was very stable, the decoherence monitored received negligible contribution from the impedance of the ring. [6] If the beam were not so stable, the experiment could not have been performed. To reveal impedance effects, the beam under study must be close to the threshold of instability, but still be stable subject to a transverse kick. In any case, we have demonstrated that Landau damping involves decoherence, but is much more than kinematic decoherence. On the other hand, simple decoherence is just a kinematic process and is different from the dynamic process of Landau damping, especially when collective effects are strong.

In order to show that decoherence is an essential ingredient of Landau damping, let us consider the Lorentzian distribution

$$
\rho\left(\omega_{\beta}\right)=\frac{\sigma}{\pi} \frac{1}{\left(\omega_{\beta}-\bar{\omega}\right)^{2}+\sigma^{2}},
$$

where $\bar{\omega}$ is the mean and $\sigma$ is the spread. We obtain by completing the upper semi-circle and picking up the poles at $\omega_{\beta}=\Omega$ and $\bar{\omega}+i \sigma$,

$$
\int_{C} d \omega_{\beta} \frac{\rho\left(\omega_{\beta}\right)}{\omega_{\beta}^{2}-\Omega^{2}}=-\frac{\Omega+i \sigma}{\Omega(\Omega-\bar{\omega}+i \sigma)(\Omega+\bar{\omega}+i \sigma)} .
$$

Substituting into Eq. (3.11), we can write

$$
\langle y(\theta, t)\rangle=\sum_{n=-\infty}^{\infty} y_{n 0} e^{i n\left(\theta-\omega_{0} t\right)} \int_{W} \frac{d \Omega}{2 \pi i} \frac{e^{-i \Omega t}\left(n \omega_{0}-\Omega\right)(\Omega+i \sigma)}{\Omega(\Omega-\bar{\omega}+i \sigma)(\Omega+\bar{\omega}+i \sigma)+i A(\Omega+i \sigma)} .
$$

The three poles in the denominator are exactly the same three solutions to the dispersion relation $\mathcal{H}(\omega)=0$. This indicates that the response of the beam center consists of three harmonic waves. Recall that the $i A /(2 \bar{\omega})$ just serves as the dynamic part of the betatron frequency shift in the absence of betatron frequency spread. We can therefore make the approximation that

$$
\frac{|A|}{2 \bar{\omega}} \ll \bar{\omega},
$$

and assume that it is of the same order as $\sigma$. Physically, Eq. (3.17) states that the dynamic frequency shift or growth rate should be much less than the mean betatron frequency and is of the order of the betatron frequency spread. Thus we can write $A=2 \bar{\omega} \epsilon$ with $|\epsilon| \sim \mathcal{O}(\sigma) \ll \bar{\omega}$. 
Here, $\epsilon$ is nothing more than the transverse impedance $Z_{1}^{\perp}$ multiplied by a positive constant. The denominator of the integrand can then be rewritten as

$$
D=\Omega[\Omega+\bar{\omega}+i(\sigma-\epsilon)][\Omega-\bar{\omega}+i(\sigma+\epsilon)]+i 2 \bar{\omega} \sigma \epsilon .
$$

The problem will be very much simplified if the last term $i 2 \bar{\omega} \sigma \epsilon$ is neglected. Keeping only the lowest order terms, the result is

$$
\begin{aligned}
&\langle y(\theta, t)\rangle=\sum_{n=-\infty}^{\infty} y_{n 0}\left[\frac{i n \omega_{0} \sigma}{\bar{\omega}^{2}} e^{i n\left(\theta-\omega_{0}\right) t}+\right. \\
&+\frac{\bar{\omega}-n \omega_{0}}{2 \bar{\omega}} e^{i n \theta-i\left(n \omega_{0}+\bar{\omega}\right) t} e^{-(\sigma+\epsilon) t}+ \\
&\left.\quad+\frac{\bar{\omega}+n \omega_{0}}{2 \bar{\omega}} e^{i n \theta-i\left(n \omega_{0}-\bar{\omega}\right) t} e^{-(\sigma-\epsilon) t}\right] .
\end{aligned}
$$

The first term is just an harmonic wave with a small frequency perturbation $\sim-2 \sigma \epsilon / \bar{\omega}$ which we have neglected. This term comes from those particles with $\omega_{\beta}=0$, because of the peculiar behavior of $\rho(0) \neq 0$ in the Lorentzian distribution. Thus, this term should not be there in a more realistic distribution. The second term corresponds to the upper betatron sidebands of all harmonics, positive and negative, while the third term corresponds to the lower sidebands. Since upper and lower sidebands will give the same physics, we can concentrate on the second term only. When $n \omega+\bar{\omega}>0$, the harmonic waves are either fast waves or super-slow waves and $\operatorname{Re} Z_{1}^{\perp}>0$. Thus $\operatorname{Re} \epsilon>0$, which enhances the kinematic decoherence rate $\sigma$. When $n \omega+\bar{\omega}<0$, we are dealing with slow waves. Since $\operatorname{Re} Z_{1}^{\perp}<0$, so is $\operatorname{Re} \epsilon$, implying that a transverse impedance cancels partly the decoherence rate. As the transverse impedance increases, the slow waves start to grow eventually when $\sigma+\mathcal{R} e \epsilon<0$ and the beam becomes unstable.

The Lorentzian distribution has been chosen here in favor of the more common Gaussian, because the former leads to a solution with a finite number of terms corresponding to a finite number of zeroes in the dispersion relation. On the other hand, a Gaussian distribution will lead to an infinite number of terms in the solution. Just retaining the term with the highest growth sometimes may not be meaningful because an infinite sum may lead to anything.

Let us apply the solution to Experiment E778. In 1987, E778 was performed with beam intensity $N_{b}=0.5 \times 10^{10}$ per bunch, rms bunch length $\sigma_{\ell} \sim 15 \mathrm{~cm}$, rms momentum spread $\sigma_{\delta}=1.5 \times 10^{-4}$, energy $E_{0}=150 \mathrm{GeV}$, and betatron tune $\nu_{\beta} \sim 20$. With a chromaticity of $\xi=10$ units, the betatron tune spread is $\sigma_{\nu_{\beta}}=\xi \sigma_{\delta}=1.5 \times 10^{-3}$, or the rms decoherence time is 670 turns. The Tevatron has a mean radius of $1 \mathrm{~km}$. For an exponential decay corresponding to the Lorentzian distribution in Eq. (3.14), the rms decay time is $\sqrt{2} / \sigma$. Thus we set $\sigma=\sqrt{2} \sigma_{\nu_{\beta}} \omega_{0}=450 \mathrm{~s}^{-1}$. The peak current $I_{0}=e N_{b} \beta c /\left(\sqrt{2 \pi} \sigma_{\ell}\right)=0.64 \mathrm{~A}$

\footnotetext{
'Knowing that this solution for $\Omega$ is much smaller than $\bar{\omega}$, we can set the second and third $\Omega$ 's on the right side of Eq. (3.18) to zero. Then it is easy to find $\Omega=i 2 \sigma \epsilon / \bar{\omega}$ to be second order in $\sigma$ or $\epsilon$.
} 
should be used in Eq. (3.2). The transverse impedance was about $Z_{1}^{\perp}=2 \mathrm{M} \Omega / \mathrm{m}$ according to the estimation in Ref. [7] from $\sim 1 \mathrm{MHz}$ to $\sim 1 \mathrm{GHz}$. We therefore obtain

$$
\epsilon=\frac{e I_{0} c Z_{1}^{\perp}}{4 \pi E_{0} \nu_{\beta}}=10.2 \mathrm{~s}^{-1}
$$

or a growth time of $\omega_{0} / \epsilon=2.9 \times 10^{4}$ revolution turns. We can therefore conclude safely that the effect of coupling impedance to the observed decoherence rate is negligible.

\section{Longitudinal Decoherence}

If decoherence and Landau damping are two completely independent mechanisms, there should be a knob with which we can turn off decoherence or Landau damping in a beam interacting with a coupling impedance. In this way, either process can be studied separately. Let us investigate whether the energy-offset distribution in a beam can serve as such a knob. In the longitudinal instabilities of a coasting beam, we learn that the beam transfer function involves the derivative of the energy distribution. In other words, there will not be any Landau damping in a flat distribution. On the other hand, decoherence appears to take place via any energy distribution. It may therefore be difficult to visualize the involvement of decoherence in Landau damping. In fact, this argument is incorrect. It is easy to show that decoherence of a coasting beam also requires the derivative of the energy distribution. [8]

Let us choose as canonical variables the energy offset $\Delta E$ and the azimuthal angle around the ring $\theta=s / R$, where $s$ is measured along the on-momentum orbit and $R$ is the mean radius of the ring. The distribution that is normalized to unity can be written as

$$
f(\theta, \Delta E ; t)=f_{0}(\Delta E)+f_{1}(\theta, \Delta E ; t),
$$

where $f_{0}(\Delta E)$ is the stationary distribution of a coasting beam which must be a function of $\Delta E$ only. The perturbation part of the distribution can be obtained by adding a harmonic deviation

$$
\delta E(\theta)=\widehat{\delta E} \cos k \theta
$$

at time $t=0$. We therefore have at $t=0+$,

$$
f(\theta, \Delta E ; 0+)=f_{0}(\Delta E-\widehat{\delta E} \cos k \theta)=f_{0}(\Delta E)-\frac{d f_{0}}{d \Delta E} \widehat{\delta E} \cos k \theta
$$

A particle moves according to $\theta=\theta^{\prime}+\omega_{0} t$ where $\theta^{\prime}$ is the position at $t=0$ and $\omega_{0}$ is the revolution angular velocity. Thus the perturbation distribution at time $t>0$ is

$$
f_{1}(\theta, \Delta E ; t)=-\frac{d f_{0}}{d \Delta E} \widehat{\delta E} \cos \left(k \theta-k \omega_{0} t\right)
$$

The perturbation part of the current recorded at $\theta_{0}$ jumps from zero at $t<0$ to

$$
I_{1}(\theta, t)=\frac{e N}{2 \pi} \int \omega_{0} f_{1}(\theta, \Delta E ; t) d \Delta E=-\frac{e N \widehat{\delta E}}{2 \pi} \int \frac{d f_{0}}{d \Delta E} \omega_{0} \cos \left(k \theta-k \omega_{0} t\right) d \Delta E
$$


at $t>0$, where $N$ is the total number of particles in the beam. We next change the variable of integration from $\Delta E$ to $\Delta \omega_{0}$, where $\Delta \omega_{0}=\omega_{0}-\bar{\omega}_{0}$ is the deviation from the nominal revolution angular frequency $\bar{\omega}_{0}$. For this, we introduce the distribution $g_{0}\left(\Delta \omega_{0}\right)$ that is normalized to unity when integrated over $\Delta \omega_{0}$ by

$$
f_{0}(\Delta E) d \Delta E=g_{0}\left(\Delta \omega_{0}\right) d \Delta \omega_{0} .
$$

Then

$$
\frac{d f_{0}}{d \Delta E} d \Delta E=\frac{d g_{0}}{d \Delta E} d \Delta \omega_{0}=-\frac{\eta \bar{\omega}_{0}}{\beta^{2} E_{0}} \frac{d g_{0}}{d \Delta \omega_{0}} d \Delta \omega_{0},
$$

where $\eta$ is the slip factor, $E_{0}$ is the nominal particle energy, $\bar{\omega}_{0}$ is the nominal revolution angular frequency, and $\beta$ is the relativistic factor. The negative sign comes about because the revolution angular frequency $\omega_{0}$ is smaller at a larger energy offset $\Delta E$ above transition $(\eta>0)$. The perturbation part of the current recorded at location $\theta_{0}$ as a function of time becomes

$$
\begin{aligned}
I_{1}(\theta, t)=\frac{e N \eta \bar{\omega}_{0}^{2} \widehat{\delta E}}{2 \pi \beta^{2} E_{0}} \int_{-\infty}^{\infty} \frac{d g_{0}}{d \Delta \omega_{0}} & {\left[\cos \left(k \theta-k \bar{\omega}_{0} t\right) \cos k \Delta \omega_{0} t+\right.} \\
+ & \left.\sin \left(k \theta-k \bar{\omega}_{0} t\right) \sin k \Delta \omega_{0} t\right] d \Delta \omega_{0} .
\end{aligned}
$$

Since $f_{0}(\Delta E)$ is even in $\Delta E, g\left(\Delta \omega_{0}\right)$ is even in $\Delta \omega_{0}$. This indicates that $d g / d \Delta \omega_{0}$ is odd in $\Delta \omega_{0}$. Thus the cosine term vanishes leaving behind

$$
I_{1}(\theta, t)=\frac{e N \eta \bar{\omega}_{0}^{2} \widehat{\delta E}}{2 \pi \beta^{2} E_{0}} \sin \left(k \theta-k \bar{\omega}_{0} t\right) \int_{-\infty}^{\infty} \frac{d g_{0}}{d \Delta \omega_{0}} \sin k \Delta \omega_{0} t d \Delta \omega_{0} .
$$

We can identify the longitudinal shock response function in a coasting beam as

$$
G_{\|}(t)=H(t) \int_{-\infty}^{\infty} \frac{d g_{0}}{d \Delta \omega_{0}} \cos k \omega_{0} t d \omega_{0},
$$

where $H(t)$ is the Heaviside step function, because it tells us how the perturbation in the current dies away after a disturbance at $t=0$. We can now understand how longitudinal kinematic decoherence takes place, especially its involvement with the derivative of the distribution function.

To conclude this section, let us compute and plot the time evolution of the energy perturbation to the beam. With the aid of Eqs. (4.3) and (4.7), the distribution in phase space is

$$
f(\theta, \Delta E ; t)=f_{0}\left[\Delta E-\widehat{\delta E} \cos \left(k \theta-k \bar{\omega}_{0} t+\frac{k \eta \bar{\omega}_{0} t}{\beta^{2} E_{0}} \Delta E\right)\right]
$$

Let us normalize everything to the rms energy spread $\sigma_{E}$ of the unperturbed beam. The distribution observed at the $n$th revolution turn is

$$
f(\theta, \Delta E ; t)=f_{0}[\varepsilon-a \cos (k \theta+k \xi \varepsilon)],
$$


where we have used the abbreviations

$$
\varepsilon=\frac{\Delta E}{\sigma_{E}}, \quad a=\frac{\widehat{\delta E}}{\sigma_{E}}, \quad \xi=\frac{2 \pi n \eta \bar{\sigma}_{E}}{\beta^{2} E_{0}} .
$$

This distribution for the energy disturbance with second harmonic $(k=2)$ and disturbance amplitude $a=\widehat{\delta E} / \sigma_{E}=0.4$ is shown in Fig. 5 at different 'times' $\xi=0,0.31,0.62,0.93$, and 1.24. In each plot, equi-density curves are depicted at $\varepsilon=-2.0$ to 2.0 in steps of 0.5 . As an example, the equi-density curve at density $x \sigma$ 's $f_{0}(x)$ is obtained by solving for $\varepsilon(\theta)$ from the equation

$$
\varepsilon-a \cos (k \theta+k \xi \varepsilon)=x .
$$

On the right is shown the energy spread distribution of the beam observed at the azimuthal angle $\theta=\pi$ along the ring, where we have assumed a Gaussian for the unperturbed beam, or

$$
f_{0}(x)=\frac{1}{\sqrt{2 \pi}} e^{-x^{2} / 2} .
$$

In the bottom is the perturbed beam current at each $\theta$ obtained by integrating over $\Delta E$, or

$$
I(\theta, \xi)=\int_{-\infty}^{\infty} f_{0}[\varepsilon-a \cos (k \theta+k \xi \varepsilon)] d \varepsilon .
$$

We notice that at time $\xi=0+$ just after the introduction of the energy disturbance, the energy-spread distribution at $\theta=\pi$ is still Gaussian but shifted to a higher energy by the amount $a=\widehat{\delta E} / \sigma_{E}$. There is completely no change in the beam current at all because the disturbance is in the energy direction.

As time progresses, shearing occurs in the beam because of the finite slip factor $\eta$ with particles having larger energy offsets shear more than those having smaller energy offsets. As a result, the equi-density curves are no longer sinusoidal and the energy distribution deviates from Gaussian. As the maxima of one equi-density curve move away from those of the others and the beam current starts exhibiting a $\cos 2 \theta$ linear density, which possesses the largest amplitude at $\xi \approx 0.5$. As time goes on, however, the shearing becomes so pronounced that the modulations at the different energy deviations overlap. This reduces the modulation of the beam current and the $\cos 2 \theta$-behavior slowly dies down in the last two plots. This is just the static decoherence of an energy disturbance in a coasting beam. Now it is easy to see why there will not be any decoherence if the energy distribution of the unperturbed beam is flat. In that case, we can no longer talk about equi-density curves. Instead every point in the longitudinal phase space has the same density or the whole longitudinal phase space is of equi-density. A truly flat distribution does not support any energy disturbance at all and therefore we cannot talk about decoherence either. For a water-bag energy distribution, an energy disturbance occurs at the two edges of the distribution. Decoherence occurs when the two edges shear differently. 

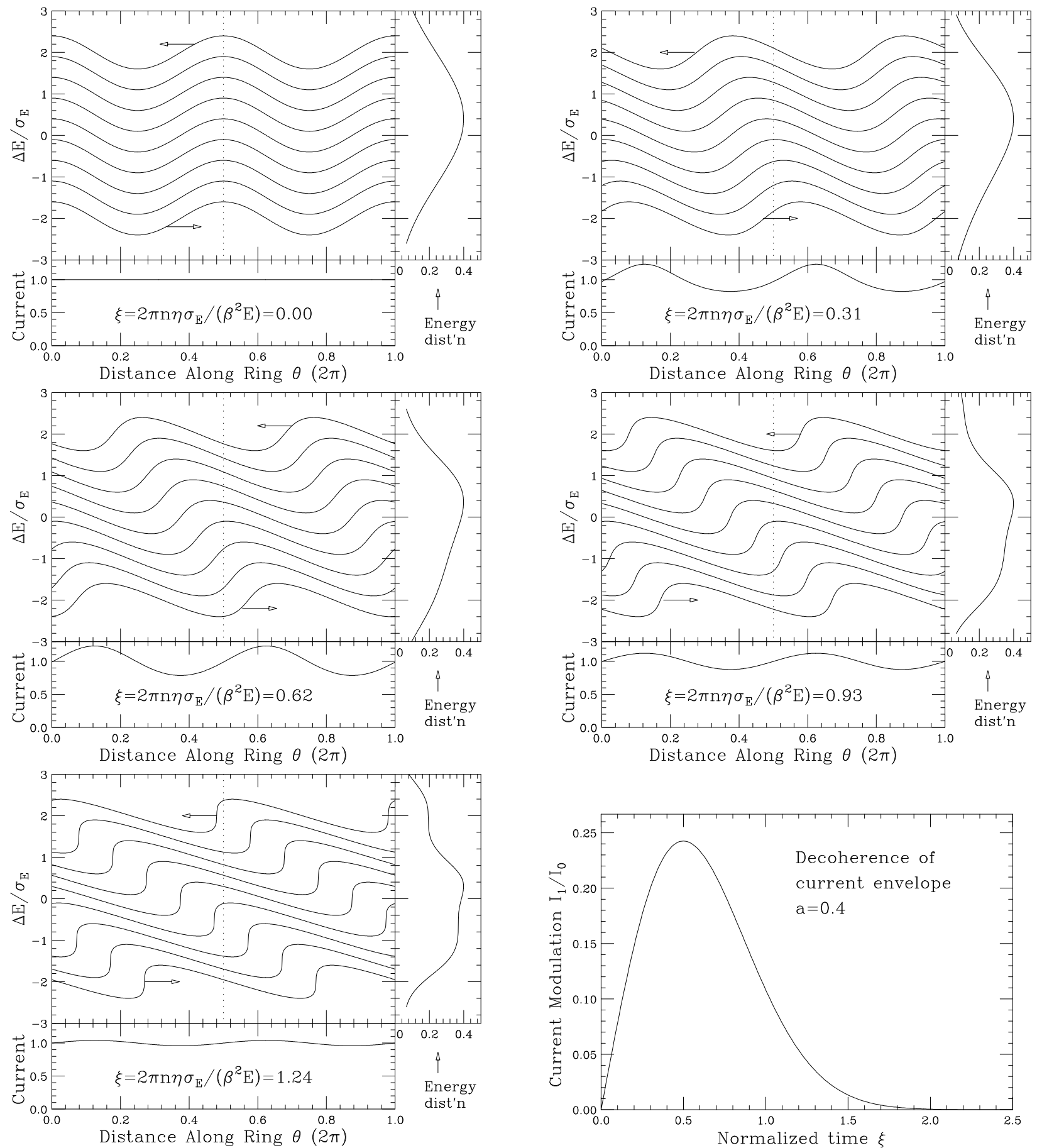

Figure 5: Illustration of longitudinal decoherence of an energy disturbance of harmonic $k=2$ and modulation amplitude $a=\widehat{\delta E} / \sigma_{E}=0.4$ in a coasting beam, where $\sigma_{E}$ is the rms energy spread of the unperturbed beam. The first five plots are at 'times' $\xi=0+, 0.31,0.62,0.93$, and 1.24. Shown in each plot are equi-density curves at $0, \pm 0.5, \pm 1.0, \pm 1.5$, and $\pm 2.0 \sigma_{E}$ 's. The energy spread distribution as recorded at azimuthal angle $\theta=\pi$ along the ring is shown at the right, with Gaussian assumed for the unperturbed. The perturbed current is shown at the bottom. The current modulation starts from zero at $\xi=0+$, goes through a maximum at $\xi=1 / k=0.5$, and rolls off like a Gaussian, as illustrated in the last plot. 
The decay of the current modulation can be derived directly from Eq. (4.5) by integrating over the revolution-frequency offset $\Delta \omega_{0}$ or the energy offset $\Delta E$. In the normalized notations, we have

$$
\frac{I_{1}(\theta, \xi)}{I_{0}}=a \sin \left(k \theta-k \bar{\omega}_{0} t\right) \int_{-\infty}^{\infty} \frac{d f_{0}}{d \varepsilon} \sin k \xi \varepsilon d \varepsilon,
$$

where $I_{0}=e N \bar{\omega} /(2 \pi)$ is the unperturbed current of the beam. For a Gaussian distribution in energy spread for the unperturbed beam, the integral can be performed exactly to give

$$
\frac{I_{1}(\theta, \xi)}{I_{0}}=-a \sin \left(k \theta-k \bar{\omega}_{0} t\right) k \xi e^{-k^{2} \xi^{2} / 2} .
$$

As monitored at location $\theta=\pi$, the envelope of the current modulation increases linearly with time, goes through a maximum at $\xi=1 / k$, and rolls off like a Gaussian, agreeing with what we observe in the numerical plots.

\section{Conclusions}

We have answered a number of questions concerning decoherence and Landau damping:

1. Decoherence is usually the smearing of the phase space because of the spread in frequency among an ensemble of oscillators. We reference this as kinematic decoherence.

2. Landau damping is the inability of an harmonic force to excite an ensemble of oscillators coherently because of the spread in frequencies among the oscillators. Only those few oscillators will continue to absorb energy from the force. For the large majority, energy is absorbed and released. Since decoherence is involved, we can reference Landau damping as dynamic decoherence.

3. Since kinematic decoherence and Landau damping are two difference processes, the damping rate for each of them should be computed and added together in a certain way. A demonstration has been made using a model of beam particles with their transverse motion coupled by the transverse impedance. A small transverse displacement of the beam excites harmonic waves in the particle beam. Depending on the sign of the real part of the impedance, these waves can be damped faster or slower than kinematic decoherence, and can even become exponentially growing in amplitude.

4. We have not been able to find a knob that can turn off decoherence or Landau damping. We have demonstrated that the longitudinal decoherence of a coasting beam also depends on the gradient of the energy-offset distribution just as in Landau damping, and is not such a knob. 


\section{Appendix}

To study the transient effects of Eq. (2.13), it is convenient to introduce two functions

$$
p(\omega)=\frac{1-\cos \omega t}{\omega} \quad \text { and } \quad d(\omega)=\frac{\sin \omega t}{\omega}
$$

as illustrated in Fig. 6. The function $p(\omega)$ always vanishes at $\omega=0$ and decays as $\omega^{-1}$ when $\omega \rightarrow \pm \infty$. It has peaks of values $\pm a t$ at $\pm b / t$, where $b=2.33$ is the root of $b=\tan (b / 2)$ and $a=2 b /\left(1+b^{2}\right)=0.725$. These peaks grow linearly with $t^{-1}$ and move closer to $\omega=0$ as $t$ increases. When $t \rightarrow \infty$, the function approaches

$$
\lim _{t \rightarrow \infty} p(\omega)=\wp \frac{1}{\omega}
$$

where $\wp$ stands for the principal value. On the other hand, $d(\omega)$ has a peak of value $t$ at $\omega=0$ and rolls off as $\omega^{-1}$ for large $\omega$, having the first zeroes at $\omega= \pm \pi / t$. As $t \rightarrow \infty$, the peak at $\omega=0$ grows linearly with $t$ while its width also shrinks inversely with $t$; the area enclosed is always $\pi$. Outside the peak, the function oscillates very fast with period $\Delta \omega \approx 2 \pi / t$. When $t \rightarrow \infty$, the function approaches

$$
\lim _{t \rightarrow \infty} d(\omega)=\pi \delta(\omega)
$$

Coming back to Eq. (2.13), as $t \gg 1 / \Delta \omega$, where $\Delta \omega$ is a measure of the width of the frequency distribution $\rho(\omega)$, all the transients die, leaving us with Eq. (2.14), the limiting displacement of the center of the ensemble of oscillators.
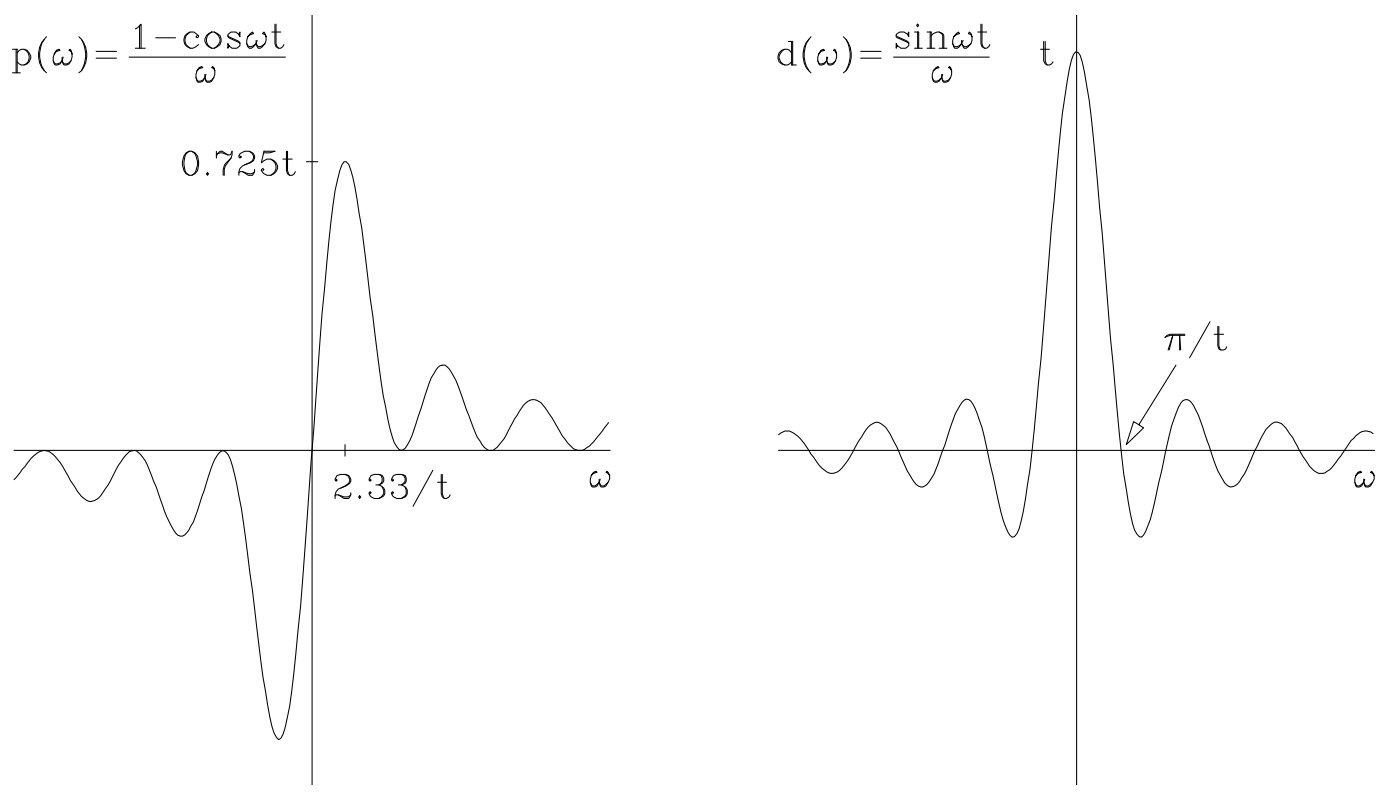

Figure 6: Plots of the functions $p(\omega)$ and $d(\omega)$ with $t$ being a parameter. As $t \rightarrow \infty$, $p(\omega) \rightarrow \wp \omega^{-1}$ and $d(\omega) \rightarrow \pi \delta(\omega)$, where $\wp$ denotes principal value. 
The derivation can be repeated with the force $A \sin \Omega t$ and the solution combined with the former to arrive at the long-term response to the force $A e^{-i \Omega t}$ :

$$
\langle y(t)\rangle=\frac{A e^{-i \Omega t}}{2 \bar{\omega}}\left[\wp \int_{-\infty}^{\infty} d \omega \frac{\rho(\omega)}{\omega-\Omega}+i \pi \rho(\Omega)\right]=\frac{A e^{-i \Omega t}}{2 \bar{\omega} \Delta \omega} R(u),
$$

where the transfer function $(\mathrm{TF})$ is defined as

$$
R(u)=f(u)+i g(u)
$$

with

$$
u=\frac{\bar{\omega}-\Omega}{\Delta \omega},
$$

and

$$
f(u)=\Delta \omega \wp \int_{-\infty}^{\infty} d \omega \frac{\rho(\omega)}{\omega-\Omega} \quad \text { and } \quad g(u)=\pi \Delta \omega \rho(\bar{\omega}-u \Delta \omega) .
$$

Here $\Delta \omega$ is a measure of the width of the frequency distribution and the parameter $u$ plays the role of the relative deviation of the frequency of the driving force from the mean frequency of the system. The transfer function is essentially the response of the particle per unit driving force. It is an important function, because it can be measured and it gives valuable information on the distribution function $\rho(\omega)$. As will be demonstrated below, when the driving force is acting on a beam inside a vacuum chamber, the transfer function contains also information on the coupling impedances of the vacuum chamber.

We can also combine the two expressions in Eq. (A.7) into one to obtain

$$
R(u)=f(u)+i g(u)=\Delta \omega \int_{-\infty}^{\infty} d \omega \frac{\rho(\omega)}{\omega-\Omega-i \epsilon} .
$$

There is a singularity in $R(u)$ when $\Omega=\omega-i \epsilon$ or $u \Delta \omega=\bar{\omega}-\omega+i \epsilon$. This implies that, if $\rho(\omega)$ is analytic, $R(u)$ is an analytic function with singularities only in the upper $u$-plane. Notice that instead of the derivation starting from the initial condition, the displacement of the center of the bunch, Eq. (A.4), can also be obtained directly by writing the force as

$$
A e^{-i(\Omega+i \epsilon) t}=A e^{-i \Omega t} e^{\epsilon t}
$$

where $\epsilon$ is an infinitesimal positive number, so that the solution becomes

$$
\langle y(t)\rangle=\frac{A e^{-i \Omega t}}{2 \bar{\omega}} \int_{-\infty}^{\infty} d \omega \frac{\rho(\omega)}{\omega-\Omega-i \epsilon}=\frac{A e^{-i \Omega t}}{2 \bar{\omega}}\left[\wp \int_{-\infty}^{\infty} d \omega \frac{\rho(\omega)}{\omega-\Omega}+i \pi \rho(\Omega)\right],
$$

which is exactly the same as Eq. (A.4). The addition of the small $\epsilon$ implies that the force in Eq. (A.9) is zero at $t=-\infty$ and increases adiabatically. It is interesting to point out the transfer function $R(u)$ is the Fourier transform of the shock-response function $G(T)$. This may shed more light on the relation between decoherence and Landau damping. 
In the study of microwave instability of a coasting beam, we can also define a longitudinal transfer function for revolution harmonic $n,[4]$

$$
R_{\|}(u)=f_{\|}(u)+i g_{\|}(u)=\left(\Delta \omega_{0}\right)^{2}\left[\wp \int d \omega_{0} \frac{\rho^{\prime}\left(\omega_{0}\right)}{\omega_{0}-\Omega / n}+i \pi \rho^{\prime}\left(\frac{\Omega}{n}\right)\right],
$$

where

$$
u=\frac{\bar{\omega}_{0}-\Omega / n}{\Delta \omega_{0}},
$$

$\rho\left(\omega_{0}\right)$ is the distribution in angular revolution frequency of the beam, with mean $\bar{\omega}_{0}$ and spread $\Delta \omega_{0}$. It differs from the transverse one by having the involvement with the gradient of the distribution function rather than the distribution function itself. It can also be shown that the longitudinal transfer function $R_{\|}(u)$ is the Fourier transform of the longitudinal shock-response function $G_{\|}(t)$ defined in Eq. (4.10). This serves as another demonstration that both Landau damping and longitudinal decoherence depend on the gradient of the distribution function in revolution frequency.

\section{References}

[1] L. D. Landau, J. Phys. USSR 10, 25 (1946); J. D. Jackson, Nucl. Energy Part C: Plasma physics 1, 171 (1960).

[2] H. G. Hereward, CERN Report 65-20 (1965).

[3] A. Hofmann, Coherent Beam Instabilities, Frontiers of Part. Beams: Intensity Limitations, Lecture Notes in Phys. 400, eds. M. Dienes, M. Month, and S. Turner (Hilton Head Island, SC, Nov. 7-14, 1990) Springer-Verlag, 1990, p. 110.

[4] A. W. Chao, Physics of Collective Beam Instabilities in High Energy Accelerators, Wiley Interscience, 1993, Chapter 5.

[5] A. Chao, D. Johnson, S. Peggs, J. Peterson, C. Saltmarsh, and L. Schachinger, R. Meller, R. Siemann, R. Talman, P. Morton, D. Edwards, D. Finley, R. Gerig, N. Gelfand, M. Harrison, R. Johnson, N. Merminga, M. Syphers, Experimental Investigation of Nonlinear Dynamics in the Fermilab Tevatron, Phys. Rev. Lett. 61, 2752 (1988); N. Merminga, D. Edwards, D. Finley, R. Gerig, N. Gelfand, M. Harrison, R. Johnson, M. Syphers, R. Meller, R. Siemann, R. Talman, P. Morton, A. Chao, T. Chen, D. Johnson, S. Peggs, J. Peterson, C. Saltmarsh, and L. Schachinger, Nonlinear Dynamics Experiment in the Tevatron, Proc. of 1989 IEEE Part. Accel. Conf., eds. F. Bennett and J. Kopta (Chicago, March 20-23, 1989), p. 1429.

[6] K. Y. Ng, Impedance Effects on Decoherence Rate of Experiment E778, SSC Report SSC-N-436, 1987. 
[7] K. Y. Ng, Impedances and Collective Instabilities of the Tevatron at Run II, Fermilab Report TM-2055, 1998.

[8] A. Hofmann, Landau Damping, CERN Accelerator School 5th Advanced Accelerator Physics Course, (Hotel Paradise, Rhodes, Greece, Sept. 20-Oct. 1, 1993), p. 275, CERN Report CERN 95-06. 